\title{
Light at night disrupts biological clocks, calendars, and immune function
}

\author{
William H. Walker II ${ }^{1}$ Jacob R. Bumgarner ${ }^{1}$ - Darius D. Becker-Krail ${ }^{1}$. Laura E. May ${ }^{1}$ Jennifer A. Liu ${ }^{1}$. \\ Randy J. Nelson ${ }^{1}$ (i)
}

Received: 26 August 2021 / Accepted: 13 October 2021 / Published online: 3 November 2021

(c) The Author(s), under exclusive licence to Springer-Verlag GmbH Germany, part of Springer Nature 2021

\begin{abstract}
Light at night is a pervasive problem in our society; over $80 \%$ of the world's population experiences significant light pollution. Exacerbating this issue is the reality that artificially lit outdoor areas are growing by $2.2 \%$ per year and continuously lit areas brighten by $2.2 \%$ each year due to the rapid growths in population and urbanization. Furthermore, the increase in the prevalence of night shift work and smart device usage contributes to the inescapable nature of artificial light at night (ALAN). Although previously assumed to be innocuous, ALAN has deleterious effects on the circadian system and circadianregulated physiology, particularly immune function. Due to the relevance of ALAN to the general population, it is important to understand its roles in disrupting immune function. This review presents a synopsis of the effects of ALAN on circadian clocks and immune function. We delineate the role of ALAN in altering clock gene expression and suppressing melatonin. We review the effects of light at night on inflammation and the innate and adaptive immune systems in various species to demonstrate the wide range of ALAN consequences. Finally, we propose future directions to provide further clarity and expansion of the field.
\end{abstract}

Keywords Light at night · Immune function · Circadian rhythms disruption · Inflammation · Melatonin

\section{Introduction}

As the Earth rotates about its axis, its position relative to the Sun results in periodic cycling of light and temperature in the form of bright warm days and dark cold nights. This constant cycling of available light is thought to have promoted life's convergent evolution of circadian rhythms-the roughly 24-h cycling of physiology and behavior that allows an organism to anticipate and adapt to this daily-changing environment. From the Latin words "circa diem," or "about a day," circadian rhythms are highly conserved across nearly all living organisms, including plants, animals (both vertebrates and invertebrates), fungi, protists, and even bacteria

This article is a contribution to the special issue on: Chronoimmunology: from preclinical assessments to clinical applications - Guest Editors: Henrik Oster \& David Ray

Randy J. Nelson

randy.nelson@hsc.wvu.edu

1 Department of Neuroscience, Rockefeller Neuroscience Institute, West Virginia University, 108 Biomedical Road, BMRC Room 370, Morgantown, WV 26506, USA
[1]. In mammals, these rhythms are driven by an endogenous self-sustaining time-keeping system that originates in the brain within an anterior hypothalamic region called the suprachiasmatic nucleus (SCN). Serving as the central pacemaker, the $\mathrm{SCN}$ receives direct photic input from the retina that entrains the rhythmic activity of its highly coupled network of neuronal and glial oscillators. This temporal information is then relayed from the SCN to a hierarchy of downstream subsidiary peripheral oscillators, synchronizing and coordinating rhythms in physiology throughout the body [2]. Temporal control even extends to the molecular level in the form of a "circadian molecular clock," whereby a highly integrated series of transcription-translation feedback loops drives rhythms in gene and protein expression to govern cellular function across time of day (reviewed in [3]). Taken together, the SCN and its complex system of subsidiary oscillators (both at the cellular and molecular level) all work together to integrate environmental light information to drive discrete rhythms in nearly all aspects of individuals' physiology and behavior-from sleep/wake activity and motivation to metabolism and immune function $[4,5]$. 
Whereas temperature, food, exercise, and even reward can all serve as "zeitgebers," or timekeepers, light remains the strongest, most potent entraining zeitgeber for the circadian system [6]. Given this characteristic, the integrity and consistency of our external light-dark cycles are critical for the maintenance of proper physiological function. Before the nineteenth century, the sun served as the primary light source for much of life on Earth, with most of the world receiving roughly $12 \mathrm{~h}$ of daylight $(50,000-100,000 \mathrm{~lx})$ and $12 \mathrm{~h}$ of relative darkness $(0.001-0.3 \mathrm{~lx})$ on average [7]. However, with the advent of the light bulb and artificial lighting systems in the late 1800 s to early 1900 s, humans began to increase their light availability, lengthening their subjective day, and ultimately changing the Earth's overall lightscape via light pollution. In a 2016 study, it was estimated that $83 \%$ of the world's population and more than $99 \%$ of the USA and Europe lives with light pollution [8]. This is a striking increase from the original 2001 study estimating $62 \%$ of the world's population lived in areas where the night sky was $>10 \%$ brighter than natural sky brightness [9]. Unfortunately, this problem will continue to worsen as artificially lit outdoor areas are growing by $2.2 \%$ per year and continuously lit areas are brightening by $2.2 \%$ each year, likely due to our rapid growths in population and urbanization [10].

Artificial light at night (ALAN) has increased at an individual behavioral level with the growing prevalence of night shift work. A 2018 survey from the Bureau of Labor Statistics estimated $16 \%$ of the USA working population worked non-daytime shiftwork, or roughly 23.6 million people [11]. This large percentage of night shift workers is likely to continue rising as healthcare continues to be the largest employing industry in the USA and among the fastest-growing sectors [12]. Whereas the average home living room ranges from 100 to $300 \mathrm{~lx}$, the average lit working environment can range from 400 to $600 \mathrm{~lx}$ - far greater intensities of light relative to the $0.3 \mathrm{~lx}$ of a full moon night sky [7].

In addition to working night shifts, smartphones, tablets, computers, TVs, and other electronic devices have all become sources of ALAN, as humans increasingly adopt technology in their daily lives. Over the past 10 years, the number of smartphone users in the USA increased from just 35\% of Americans in 2011 to nearly 85\% in 2021 [13]. Not only in the USA, but the adoption of smartphone usage has also increased significantly around the world, with more than $80 \%$ of the global population having a smartphone [14]. This widespread adoption of smart devices has undoubtedly contributed to increased ALAN, given that $95 \%$ of surveyed Americans report regular electronic usage within an hour of bedtime and $70 \%$ of adults report accessing social media before bed [14]. Although the light emitted from the average smartphone or other backlit portable electronic device may only be around $40 \mathrm{~lx}$, the emission of high intensity short-wavelength blue light from these LED and LCDs are thought to be the most impactful [15].

In mammals, the retina comprises specialized intrinsically photosensitive retinal ganglion cells (ipRGCs) that express the photosensitive pigment melanopsin and primarily serve to take in circadian photic information. This phototransduction occurs when light of a specific frequency enters the eye, activates the melanopsin in the ipRGCs, and an action potential is initiated that eventually gets transduced to the SCN via the retinohypothalamic tract [16]. Melanopsin is most strongly activated by wavelengths near the blue end of the spectrum of visible light ( 420-440 nm), and least affected by wavelengths near the red end of the spectrum $(>600 \mathrm{~nm})$ [17]. This spectral sensitivity of melanopsin is particularly relevant in the context of ALAN, in that both blue-rich white LEDs have become the predominant indoor light source and smartphone usage is heavily skewed towards blue light emission [15, 18]. Given the intrinsic blue-light sensitivity of melanopsin and the heavy proportion of high-intensity blue light emitted from artificial lighting, it is critical to consider how ALAN may be deleteriously affecting the circadian system and circadian regulated physiology.

\section{ALAN alters circadian clocks and melatonin secretion}

In nature, photic signaling from the retina to the $\mathrm{SCN}$ encodes time-of-day information about the surrounding environment. Photic signaling cues at the beginning, end, and throughout the duration of the light phase are integrated in the SCN to modify the cellular and molecular activity of local neurons and astrocytes. For example, light cues received by the SCN can directly modify the expression of clock genes. Light pulses in the dark phase enhance the expression of Clock [19], Per1 [20], and Per2 in humans [21] and rodents [22-24]. These alterations to clock genes are in turn translated into temporal neuronal, humoral, and molecular cues that synchronize peripheral clocks to the daily light-dark cycle and altering day lengths across the year.

SCN activity drives the rhythmic secretion of an important synchronizing humoral cue: melatonin. Via the modulation of sympathetic tone, SCN activity during the dark phase stimulates the secretion of melatonin from the pineal gland [25]. Unlike many other circadian rhythms, melatonin secretion is phase-independent; it is secreted at night in both nocturnal and diurnal animals. Melatonin functions to synchronize peripheral rhythms, and its duration of nocturnal secretion encodes day length, a critical feature driving seasonal rhythms [19]. A crucial function of melatonin is the modulation of the activity of the immune system (Fig. 1) [26]. 


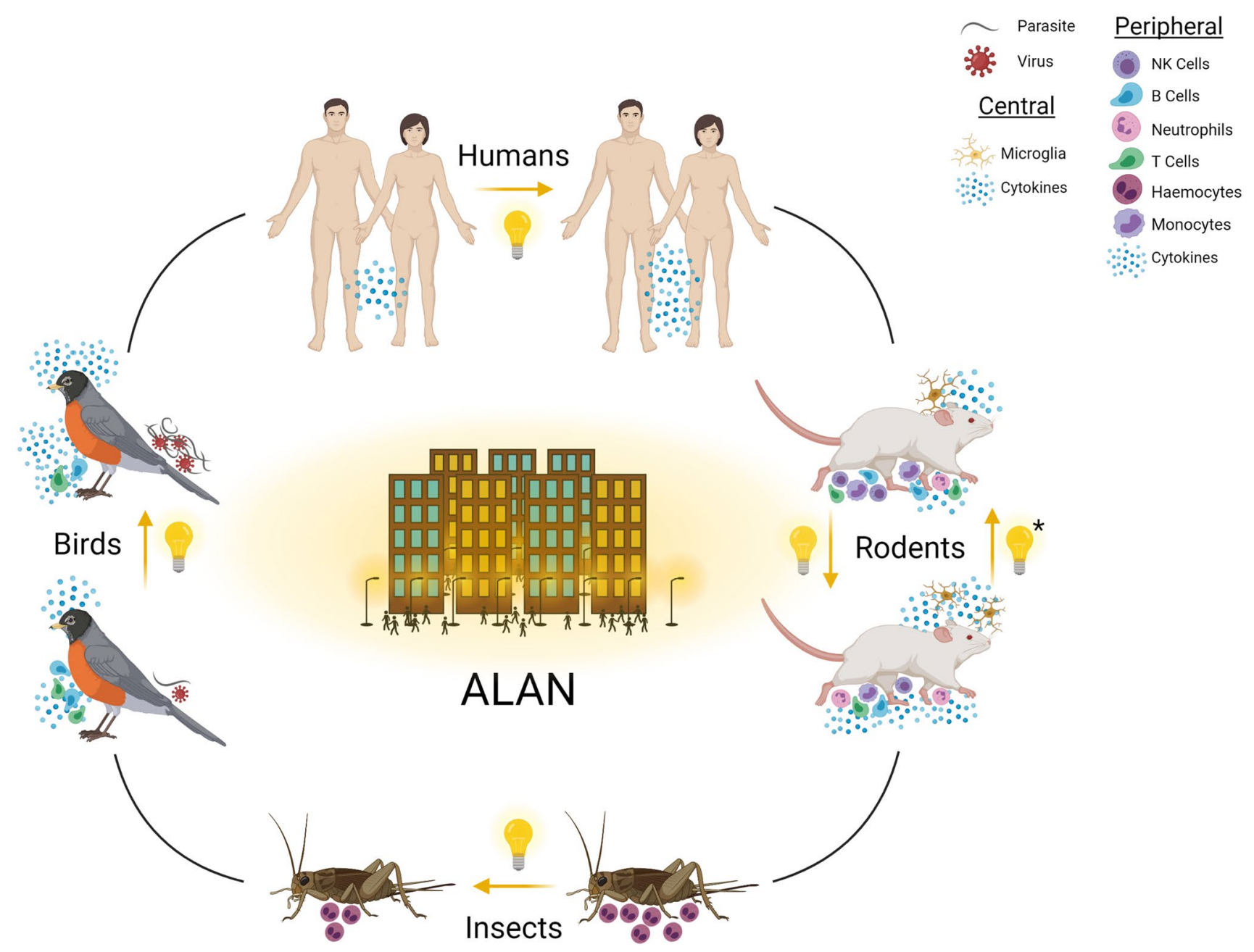

Fig. 1 ALAN perturbs the function of the immune system. *ALAN differentially affects immune function in rodents depending on the production of pineal melatonin

The immune system exhibits circadian rhythms in function that enable optimal energetic resource allocation as organisms vary their activity across the day [27]. Circadian rhythms in immune function are often phase-dependent such that they follow activity-rest patterns. For example, circadian rhythms are observed in the number of circulating leukocytes, the production of cytokines, and responses to immune challenges [28]. Importantly, the rhythmic activity of the immune system across the day is coordinated by the modulation of autonomic tone by the SCN, clock gene regulation of gene expression, and the fluctuation of humoral signaling cues, such as melatonin [26]. Melatonin acts to both synchronize immune activity across the day and modulate the activity of the immune system. Depending on the conditions in which it is secreted, melatonin can act as a proinflammatory and anti-inflammatory agent [29].

As our modern environments are flooded with ALAN, photic signaling information no longer consistently encodes time-of-day information. Because the SCN is sensitive to photic signaling during the dark phase, ALAN directly and indirectly alters clock gene loops in numerous regions of tissue. ALAN alters the production of dark phase PER1 and PER2 in the SCN [20,30,31], Clock and Cryl in the hippocampus [32], and Perl/2/3 in the pineal gland [33] of rodents. ALAN also perturbs clock gene loops outside of CNS, including altered loops in the liver, heart, adrenal glands [33], and fat [34]. These effects are also observed in other species, including mosquitos [35] and zebra finches [36].

Synchronized melatonin secretion from the pineal gland is dependent on consistent dark-phase cues from the SCN. Exposure to light suppresses melatonin secretion, thereby restricting its secretion to the dark phase [19]. However, the SCN cannot distinguish between natural and artificial lighting sources: light is light. Just as natural light, ALAN suppresses melatonin in numerous species in an intensitydependent manner. Indeed, ALAN blunts melatonin secretion in humans [37, 38], lemurs [39], finches [36, 40, 41], 
several species of fish $[42,43]$, and rodents [44]. ALAN also modifies the sensitivity of the SCN to light pulses in the night [20]. This suggests that ALAN may limit the ability of organisms to exhibit photoperiodic responses to varying day lengths across the year.

Synchronization of circadian rhythms of the immune system is dependent on organizing cues originating from the $\mathrm{SCN}$, including melatonin. Because ALAN alters clock gene loops, melatonin secretion, and numerous other synchronizing cues, its effects on behavior and physiology are numerous [45]. The consequences of ALAN on immune function are becoming increasingly evident.

\section{ALAN and innate immune function}

Optimal functioning of an organism's immune system is critical for protection and survival from illness and disease. However, increasing evidence has demonstrated that dramatic growth in population/urbanization and subsequent increase in ALAN can have deleterious consequences on an organism's ability to respond to an immune challenge. There is clear evidence that ALAN alters innate immune function; typically, ALAN impairs innate immune responses. This effect occurs across the animal kingdom among both vertebrates and invertebrates. Indeed, constant lighting reduced circulating melatonin and impaired immune function in crickets (Teleogryllus commodus) [46] Specifically, male and female crickets were reared from eggs to 5 weeks of age post-adult emergence in either a 12:12 LD cycle or $24 \mathrm{~h}$ constant lighting condition (4000 lx). Notably, invertebrates lack an adaptive immune system, but maintain a highly effective innate immune response composed of both cellular and humoral defense pathways [47, 48]. Immune function was assessed via hemocyte concentration (cellular defense pathway, i.e., phagocytosis and encapsulation), lytic activity (humoral defense pathway, i.e., capacity of antibacterial enzymes to degrade bacterial cell walls), and phenoloxidase (PO) activity (humoral defense pathway encapsulation mechanism for parasitism or infection of larger foreign body) [47, 49]. Constant lighting significantly reduced hemocyte concentrations and negatively impacted lytic activity. However, there was no effect on PO activity [46]. In a subsequent follow-up study, the authors assessed the effects of dim light at night on innate immune function in male and female crickets [50]. Crickets were reared in either a 12:12 LD cycle (2600 lx:0 lx) or one of three dim light at night conditions (2600 lx: 1, 10, or $100 \mathrm{~lx}$ at night). Similar to the previous study, immune function was assayed at 4 weeks of age post-adult emergence via hemocyte concentration, lytic activity, and PO activity. Housing crickets in dim light at night significantly reduced hemocyte concentrations at 4 weeks irrespective of the specific dim light at night condition. Lytic activity and PO activity were not significantly affected [50]. Together, these studies demonstrate that ALAN can have detrimental consequences on invertebrate innate immune function, particularly, hemocyte concentrations.

Avian research comprises a large portion of studies assessing the effects of ALAN on innate immune function in vertebrates. Similar to invertebrates, ALAN primarily has a negative impact on innate immune function. For example, exposure to ALAN impaired the innate immune response in wild great tit nestlings (Parus major) [51]. Indeed, nestlings exposed to $3 \mathrm{~lx}$ ALAN for seven consecutive nights and immune challenged via a lipopolysaccharide injection, demonstrated lower haptoglobin and higher nitric oxide levels following the immune challenge compared with dark-night nestlings [51]. This effect was likely a result of reduced melatonin, as ALAN treated birds had 49\% lower melatonin concentrations relative to dark night birds. In contrast, exposure to ALAN increased innate immune activity during development in king quail (Excalfactoria chinensis) [52]. Three-week-old king quail were housed in either 16:8 LD conditions or 16:8 ALAN conditions (0.3 lx light at night) for 6 weeks. Blood samples were taken weekly to determine ex vivo bacterial-killing capacity. Housing quail in ALAN transiently increased the bacterial killing capacity at 4 weeks for females and 6 weeks for males [52]. However, whether the transient increases in innate immune activity has a positive or negative effect on fitness remains to be determined. Ecological and laboratory studies suggest that ALAN has a negative effect on fitness. Indeed, ALAN amplified the seasonal relapse of haemosporidian parasites in songbirds [53]; male juncos (Junco hyemalis) were captured and housed on an ecologically relevant photoperiod. Juncos were housed in either dark night or ALAN conditions (2.5 lx). Notably, ALAN increased the presence of parasites within the blood with significant peaks in spring and autumn that were not seen in juncos housed in dark night conditions [53]. Similarly, ecological studies in adult great tits (Parus major) have demonstrated that birds roosting in field sites exposed to white light at night had a higher probability of malaria infection relative to their dark night counterparts [54].

Studies in rodents have reported conflicting conclusions regarding the effects of ALAN on innate immune function. ALAN impaired the daily variation of circulating immune cells in male Wistar rats [55]. Primarily, ALAN (2 lx) significantly reduced blood monocyte concentrations after 2 weeks of ALAN (ZT9) and altered the daily variation in blood monocyte concentrations following 5 weeks of housing in ALAN (ZT9 and ZT21). Similarly, in male F344 rats, a single night of housing in ALAN (200 lx) suppressed the nocturnal increase in number and cytotoxic activity of splenic NK cells [56]. In contrast, CD-1 mice house in constant illumination of $750 \mathrm{~lx}$ ALAN for 4 weeks demonstrated 
significantly increased granulocytic CD11 $b^{+}$Ly $6 G^{\text {high }}$ and monocytic $\mathrm{CD} 11 \mathrm{~b}^{+} \mathrm{CD} 49^{\mathrm{d}+}$ myeloid subsets within the spleen [57]. ALAN increased the plasma bacterial killing capacity in male Nile grass rats (Arvicanthis niloticus) housed in $5 \mathrm{~lx}$ of light at night for 3 weeks [58]. In contrast, 4 weeks of 5 lx ALAN reduced the blood plasma bacterial killing capacity in Siberian hamsters (Phodopus sungorus) following an LPS challenge (16:8 LD) [59]. Most striking is the ability of ALAN before conception to attenuate the offspring's innate immune response [60]. Male and female Siberian hamsters were exposed to either dark nights or ALAN (5 lx) for 8 weeks. Hamsters were paired and mated, and thereafter housed in dark nights. Paternal pre-conception ALAN exposure reduced serum bacterial killing capacity, demonstrating that ALAN can have transgenerational effects on innate immunity [60]. Notably, the effects of ALAN are not specific to peripheral immune function as mice exposed to dim light at night display an exaggerated CNS inflammatory response to lipopolysaccharide [61]. Indeed male Swiss Webster mice housed in 5 lx ALAN for 4 weeks demonstrated elevated microglia pro-inflammatory cytokine expression (TNF and IL-6) following LPS administration [61]. Similar effects are seen in female C57B16 mice with exposure to ALAN during development and subsequent challenge with LPS in adulthood [62]. Furthermore, housing male Swiss Webster mice in 5 lx ALAN for 7 days following cardiac arrest resulted in an increase in CNS proinflammatory cytokine expression, elevated Iba1 immunoreactivity (a marker for microglial activation with concurrent cytokine data), and reduced survival [63].

The discrepancies in rodent studies may reflect differences in species, diurnality, or melatonin secretion. The data demonstrate that in nocturnal species with robust pineal melatonin rhythms (rats and hamsters), ALAN suppresses innate immune function, even transgenerational innate immune function. In contrast, in both nocturnal rodents (i.e., most mice) lacking robust pineal melatonin rhythms and in diurnal rodents (i.e., grass rats), ALAN may enhance aspects of the innate immune system. Future studies should continue to examine the effects of ALAN on innate immune function to provide further clarity. In particular, studies should focus on diurnal rodents with robust pineal melatonin rhythms and should expand their study population to include female rodents, which are dramatically understudied in the current literature.

\section{ALAN and adaptive immune function}

Unlike innate immune function, few studies have examined the effects of ALAN on adaptive immune function outside of rodents. However, the few avian studies have concluded that ALAN suppresses adaptive immune function. Constant lighting for 8 weeks suppresses total leukocyte and lymphocyte counts in male and female Indian jungle bush quail (Perdicula asiatica) during the inactive phase [64]. Furthermore, in female Japanese quail (Coturnix japonica), constant lighting (700 1x) for 3 weeks before an immune challenge suppressed both cellular immunity, as measured by a cutaneous basophil hypersensitivity reaction to phytohemagglutinin, and humoral immune response, as measured by antibody titers to a previous inoculation of Chukar red blood cell suspension [65]. Administration of melatonin during their inactive phase enhanced both cellular and humoral immunity in quail housed in constant lighting [65]. Notably, the effects of ALAN-induced suppression of the adaptive immune system are likely functionally relevant. ALAN (8 lx) extends the infectious period of West Nile virus (WNV) in male and female house sparrows (Passer domesticus) [66]. ALAN exposed sparrows maintained adequate viral titers for transmissibility 2 days longer than dark night-housed counterparts. Particularly relevant to the current COVID-19 pandemic, mathematical modeling demonstrated that ALAN could increase the outbreak potential of WNV by approximately $41 \%$ due to the extended time of transmissibility [66].

Similar to rodent studies on innate immune function following ALAN exposure, rodent studies on adaptive immune function can be conflicting at times. Mizutani and colleagues (2017) sought to examine the effects of ALAN on immune tolerance. Previous studies have demonstrated that antigen sensitization during early development results in tolerance of the antigen-specific allergic response in adulthood [67]. $\mathrm{Balb} / \mathrm{C}$ mice housed in constant lighting from gestation or birth until 7 weeks of age demonstrated significantly reduced immune tolerance to 2,4,6-trinitro-1-chlorobenzene (TNCB) [68]. The authors concluded that this effect is likely due to the reduced T-reg cells present in the inflamed skin and draining lymph nodes of mice housed in constant light. Similarly, male Nile grass rats housed in 5 lx ALAN for 3 weeks increased their delayed-type hypersensitivity (DTH) response (T-cell mediated immune response) and increased antibody production following inoculation with keyhole lymphocyte hemocyanin (KLH) [58]. Furthermore, prior exposure to ALAN impaired dermal wound healing in female C57BL/6 mice. Mice housed in ALAN prior to wounding demonstrated significantly larger wound sizes between days 5 and 9 . Notably, these days correspond to the initiation of lymphocyte trafficking into the wound (day 5), the peak of lymphocytes within the wound (day 7), and the resolution of lymphocytes ( day 10) [69].

The effects of ALAN exposure on adaptive immune function in photoperiodic rodents are straightforward. ALAN suppresses adaptive immune function. For example, male Siberian hamsters housed in ALAN (5 lx) for 4 weeks demonstrated significantly reduced DTH response [59]. Furthermore, the DTH response was significantly reduced 
following acute (6 $\mathrm{h}$ of restraint) or chronic ( 2 weeks of restraint $6 \mathrm{~h}$ per day) stress in male Siberian hamsters housed in ALAN (5 lx) for 4 weeks or 8 weeks, respectively [70, 71]. Most striking is the ability of ALAN to have transgenerational effects on the adaptive immune system [72]; male and female Siberian hamsters were housed in either dark nights or ALAN (5 lx) for 9 weeks. The hamsters were then paired and mated in a full factorial design and thereafter housed in dark nights. Exposure of dams to ALAN reduced the DTH responses in male offspring; paternal and maternal exposure to ALAN reduced the DTH response in female offspring [72]. Together these data demonstrate that ALAN suppresses adaptive immune function in avians and photoperiodic rodents (i.e., hamsters). In contrast, ALAN tends to enhance adaptive immune function in both rodents lacking robust pineal melatonin rhythms (i.e., most mice) and in diurnal rodents (i.e., grass rats).

\section{ALAN and inflammation}

Numerous studies have examined the influence of ALAN on general inflammation in the absence of immune challenge. As a result of direct and indirect clock-gene regulation of cytokine transcription [73, 74], central and peripheral immune cells exhibit daily rhythms of cytokine production and secretion $[28,75]$. Perturbation of clock gene loops by circadian rhythm disruption or genetic manipulations can induce heightened immune activation and production of pro-inflammatory cytokines, even in the absence of immune challenge [76].

ALAN consistently elevates basal inflammation. The majority of research demonstrating this effect has been conducted in rodents, with several corroborating studies in other species. Female Siberian hamsters exposed to ALAN for 4 weeks exhibited elevated hippocampal TNF, an effect which was associated with heightened depressivelike behavior [77]. Male mice exposed to 5 lx of ALAN also exhibited elevated hippocampal TNF and Il-6 [78] and medulla Il-6 [79]. In an extreme example, exposure to only 3 nights of $5 \mathrm{~lx}$ of light elevated hippocampal Il- $1 \beta$ in female mice, demonstrating that ALAN can have both rapid and prolonged effects on inflammation [32]. Peripherally, ALAN elevates hepatic MAC1 and TNF [34]. In contrast, one study observed reduced kidney $\mathrm{Ccl} 2$ expression following 2 weeks of ALAN [80].

The effects of ALAN on heightened inflammation or heightened immune tone are noted in other species, including humans. In an at-home randomized crossover study, humans that slept with $40 \mathrm{~lx}$ of light in their bedrooms were observed to have elevated plasma high-sensitivity C-reactive protein concentrations [81]. Similarly, great tit nestlings exposed to only two nights of ALAN exhibited elevated plasma haptoglobin levels [82]. ALAN also elevated nocturnal Il-6 expression in the hippocampus of zebra finch [41]. Taken together, the current evidence indicates that ALAN disrupts both central and peripheral clock gene loops, which in turn elevates the production of pro-inflammatory cytokines. However, from these single time-point results alone, it is not apparent whether ALAN is simply elevating baseline inflammation across the entire day or is disrupting cytokine rhythms. Instead, ALAN may be shifting, altering the amplitude, or abolishing the rhythmic production of central and peripheral cytokines.

To date, only one study has examined the effect of ALAN on the rhythmic production of cytokines [41]. Female and male zebra finch were group-housed under 12:12 light-dark cycles with either dark nights or dim blue ALAN exposure at 3 lx [41]. Centrally, ALAN abolished Il-6 and Il-10 rhythms in the hypothalamus and Il- $1 \beta$ rhythms in the hippocampus. ALAN abolished Il- $1 \beta$ and Il- 6 rhythms in the spleen, blunted Il- $1 \beta$ rhythms in the liver, and phase-shifted Il- $1 \beta$ and elevated the amplitude of Il-6 rhythms in the fat. Differential effects of ALAN on Il-10 were observed. ALAN reduced Il-10 expression across numerous time points in the hippocampus and hypothalamus, but elevated Il-10 expression in the nidopallium across the day. These results suggest that the effects of ALAN not only alter the expression of pro-inflammatory cytokines, but the effects of ALAN on inflammation differ between tissue regions and even nuclei. Additionally, ALAN can alter cytokine production rhythms, including the abolishment of rhythms, alteration of amplitudes, and alteration of mean expression across the day [41]. To further elucidate the complex dynamics between circadian rhythm disruption and altered immune function, future work should examine the effects of ALAN on cytokine production rhythms across the day rather than single time points.

\section{Conclusions}

Several key aspects of the immune system are governed by circadian rhythms. Here, we highlight the relationship between ALAN exposure and altered immune function. Specifically, ALAN exposure detrimentally affects both the innate and adaptive immune function in invertebrates, birds, and rodents with robust pineal melatonin rhythms. Conversely, in diurnal rodents and species without robust pineal melatonin rhythms, the data demonstrate that ALAN may enhance aspects of the innate and adaptive immune systems. Nocturnal rodents remain the most prevalent species used in studies investigating the effects of nighttime light exposure on immune function. However, diurnal rodents exhibit vastly different secretion patterns for both hormonal and immune parameters compared to nocturnal species [83]. 
Due to the diurnality of humans, future studies should focus on diurnal rodents with robust pineal melatonin rhythms. However, this is not to say that melatonin is the only mediator of ALAN effects, as studies have demonstrated melatonin independent effects (i.e., altered clock gene rhythms in species lacking pineal melatonin) [31, 32]. In addition, most studies reviewed focus primarily on males. Thus, expanding the field to examine the effects of ALAN on females should be an area of concentration. Lastly, few studies examined report the time of day of tissue collection or immune challenge. Given the circadian regulation of immune function and the renewed call for time of day reporting in all fields [84], future studies should take care to report time of day to allow for increased understanding and replication.

Author contribution WHWII: designed, wrote, and edited the manuscript, and designed the figure. JRB, DDBK, LEM, JAL: wrote and edited the manuscript and designed the figure. RJN: designed, wrote, and edited the manuscript.

Funding The authors were supported by grants from NINDS (R01NS092388 to R.J.N.), NCCIH (R21AT011238-01 to R.J.N), and NIGMS award number 5U54GM104942. The content is solely the responsibility of the authors and does not necessarily represent the official views of the National Institutes of Health.

\section{Declarations}

Conflict of interest The authors declare no competing interests.

\section{References}

1. Bhadra U, Thakkar N, Das P, Pal Bhadra M (2017) Evolution of circadian rhythms: from bacteria to human. Sleep Med 35:4961. https://doi.org/10.1016/j.sleep.2017.04.008

2. Hastings MH, Maywood ES, Brancaccio M (2019) The mammalian circadian timing system and the suprachiasmatic nucleus as its pacemaker. Biology (Basel) 8:13. https://doi.org/10.3390/ biology 8010013

3. Partch CL, Green CB, Takahashi JS (2014) Molecular architecture of the mammalian circadian clock. Trends Cell Biol 24:90-99

4. Antle MC, Silver R (2016) Circadian insights into motivated behavior. Curr Top Behav Neurosci 27:137-169. https://doi.org/ 10.1007/7854\_2015\_384

5. Carroll RG, Timmons GA, Cervantes-Silva MP et al (2019) Immunometabolism around the clock. Trends Mol Med 25:612625. https://doi.org/10.1016/j.molmed.2019.04.013

6. Challet E, Pévet P (2003) Interactions between photic and nonphotic stimuli to synchronize the master circadian clock in mammals. Front Biosci 8:s246-s257. https://doi.org/10.2741/1039

7. Gaston KJ, Bennie J, Davies TW, Hopkins J (2013) The ecological impacts of nighttime light pollution: a mechanistic appraisal. Biol Rev 88:912-927. https://doi.org/10.1111/brv.12036

8. Falchi F, Cinzano P, Duriscoe D et al (2016) The new world atlas of artificial night sky brightness. Sci Adv 2:e1600377-e1600377. https://doi.org/10.1126/sciadv.1600377
9. Cinzano P, Falchi F, Elvidge CD (2001) The first world atlas of the artificial night sky brightness. Mon Not R Astron Soc 328:689-707. https://doi.org/10.1046/j.1365-8711.2001. 04882.x

10. Kyba CCM, Kuester T, de Miguel A et al (2017) Artificially lit surface of Earth at night increasing in radiance and extent. Sci Adv 3:e1701528. https://doi.org/10.1126/sciadv.1701528

11. Job flexibilities and work schedules summary. https://www.bls. gov/news.release/flex2.nr0.htm. Accessed 17 Aug 2021

12. Health care still largest u.s. employer. https://www.census.gov/ library/stories/2020/10/health-care-still-largest-united-statesemployer.html. Accessed 17 Aug 2021

13. Demographics of mobile device ownership and adoption in the united states I Pew Research Center. https://www.pewresearch. org/internet/fact-sheet/mobile/. Accessed 17 Aug 2021

14. Bhat S, Pinto-Zipp G, Upadhyay H, Polos PG (2018) “To sleep, perchance to tweet": in-bed electronic social media use and its associations with insomnia, daytime sleepiness, mood, and sleep duration in adults. Sleep Heal 4:166-173. https://doi.org/10. 1016/j.sleh.2017.12.004

15. Oh JH, Yoo H, Park HK, Do YR (2015) Analysis of circadian properties and healthy levels of blue light from smartphones at night. Sci Rep 5:11325. https://doi.org/10.1038/srep11325

16. Berson DM, Dunn FA, Takao M (2002) Phototransduction by retinal ganglion cells that set the circadian clock. Science 80(295):1070-1073. https://doi.org/10.1126/science.1067262

17. Brown RL, Robinson PR (2004) Melanopsin-shedding light on the elusive circadian photopigment. Chronobiol Int 21:189-204. https://doi.org/10.1081/cbi-120037816

18. Touitou Y, Point S (2020) Effects and mechanisms of action of light-emitting diodes on the human retina and internal clock. Environ Res 190:109942. https://doi.org/10.1016/j.envres.2020. 109942

19. Wood S, Loudon A (2014) Clocks for all seasons: unwinding the roles and mechanisms of circadian and interval timers in the hypothalamus and pituitary. J Endocrinol 222:R39-R59

20. Shuboni D, Yan L (2010) Nighttime dim light exposure alters the responses of the circadian system. Neuroscience 170:1172-1178. https://doi.org/10.1016/j.neuroscience.2010.08.009

21. Cajochen C, Jud C, Münch M et al (2006) Evening exposure to blue light stimulates the expression of the clock gene PER2 in humans. Eur J Neurosci 23:1082-1086

22. Shigeyoshi Y, Taguchi K, Yamamoto S et al (1997) Light-induced resetting of a mammalian circadian clock is associated with rapid induction of the mPer1 transcript. Cell 91:1043-1053. https://doi. org/10.1016/s0092-8674(00)80494-8

23. Albrecht U, Sun ZS, Eichele G, Lee CC (1997) A differential response of two putative mammalian circadian regulators, mper1 and mper2, to light. Cell 91:1055-1064. https://doi.org/10.1016/ s0092-8674(00)80495-X

24. Miyake S, Sumi Y, Yan L et al (2000) Phase-dependent responses of Per1 and Per2 genes to a light-stimulus in the suprachiasmatic nucleus of the rat. Neurosci Lett 294:41-44. https://doi.org/10. 1016/s0304-3940(00)01545-7

25 Colwell CS, Witkovsky P, Silver R (2015) The suprachiasmatic nucleus (SCN): critical points. In: Colwell CS (ed) Circadian Medicine. Wiley Blackwell Inc., Hoboken, pp 37-55

26. Esquifino AI, Pandi-Perumal SR, Cardinali DP (2004) Circadian organization of the immune response: a role for melatonin. Clin Appl Immunol Rev 4:423-433

27. Logan RW, Sarkar DK (2012) Circadian nature of immune function. Mol Cell Endocrinol 349:82-90. https://doi.org/10.1016/j. mce.2011.06.039

28. Scheiermann C, Kunisaki Y, Frenette PS (2013) Circadian control of the immune system. Nat Rev Immunol 13:190-198. https://doi. org/10.1038/nri3386 
29. Hardeland R (2019) Aging, melatonin, and the pro-and antiinflammatory networks. Int J Mol Sci 20(5):1223

30. Bedrosian TA, Galan A, Vaughn CA et al (2013) Light at night alters daily patterns of cortisol and clock proteins in female siberian hamsters. J Neuroendocrinol 25:590-596. https://doi.org/10. 1111/jne. 12036

31. Fonken LK, Aubrecht TG, Meléndez-Fernández OH et al (2013) Dim light at night disrupts molecular circadian rhythms and increases body weight. J Biol Rhythms 28:262-271. https://doi. org/10.1177/0748730413493862

32. Walker WH, Borniger JC, Gaudier-Diaz MM et al (2020) Acute exposure to low-level light at night is sufficient to induce neurological changes and depressive-like behavior. Mol Psychiatry 25:1080-1093. https://doi.org/10.1038/s41380-019-0430-4

33. Cailotto C, Lei J, van der Vliet J et al (2009) Effects of nocturnal light on (clock) gene expression in peripheral organs: a role for the autonomic innervation of the liver. PLoS One 4:e5650

34. Fonken LK, Lieberman RA, Weil ZM, Nelson RJ (2013) Dim light at night exaggerates weight gain and inflammation associated with a high-fat diet in male mice. Endocrinology 154:3817-3825. https://doi.org/10.1210/en.2013-1121

35 Honnen, Kypke, Hölker, Monaghan (2019) Artificial light at night influences clock-gene expression, activity, and fecundity in the mosquito culex pipiens f. molestus. Sustainability 11:6220. https://doi.org/10.3390/su11226220

36. Batra T, Malik I, Kumar V (2019) Illuminated night alters behaviour and negatively affects physiology and metabolism in diurnal zebra finches. Env Pollut 254:112916. https://doi.org/10.1016/j. envpol.2019.07.084

37. Higuchi S, Nagafuchi Y, Lee S, Harada T (2014) Influence of light at night on melatonin suppression in children. J Clin Endocrinol Metab 99:3298-3303

38. Lewy AJ, Wehr TA, Goodwin FK et al (1980) Light suppresses melatonin secretion in humans. Science 80(210):1267-1269

39. Le Tallec T, Théry M, Perret M (2016) Melatonin concentrations and timing of seasonal reproduction in male mouse lemurs (Microcebus murinus) exposed to light pollution. J Mammal 97:753-760

40. Moaraf S, Vistoropsky Y, Pozner T et al (2020) Artificial light at night affects brain plasticity and melatonin in birds. Neurosci Lett 716:134639

41. Mishra I, Knerr RM, Stewart AA et al (2019) Light at night disrupts diel patterns of cytokine gene expression and endocrine profiles in zebra finch (Taeniopygia guttata). Sci Rep 9:15833. https://doi.org/10.1038/s41598-019-51791-9

42. Brüning A, Kloas W, Preuer T, Hölker F (2018) Influence of artificially induced light pollution on the hormone system of two common fish species, perch and roach, in a rural habitat. Conserv Physiol 6:coy016

43. Brüning A, Hölker F, Franke S et al (2018) Influence of light intensity and spectral composition of artificial light at night on melatonin rhythm and mRNA expression of gonadotropins in roach Rutilus rutilus. Fish Physiol Biochem 44:1-12

44. Agbaria S, Haim A, Fares F, Zubidat AE (2019) Epigenetic modification in 4T1 mouse breast cancer model by artificial light at night and melatonin-the role of DNA-methyltransferase. Chronobiol Int 36:629-643. https://doi.org/10.1080/07420528.2019. 1574265

45 Bumgarner JR, Nelson RJ (2021) Light at night and disrupted circadian rhythms alter physiology and behavior. Integr Comp Biol 61(3):1160-1169

46. Durrant J, Michaelides EB, Rupasinghe T et al (2015) Constant illumination reduces circulating melatonin and impairs immune function in the cricket Teleogryllus commodus. PeerJ 3:e1075. https://doi.org/10.7717/PEERJ.1075

47. Beckage NE (ed) (2011) Insect immunology. Academic press
48. Rosales C (2017) Cellular and molecular mechanisms of insect immunity. Insect Physiol Ecol. https://doi.org/10.5772/67107

49. Ribeiro C, Brehélin M (2006) Insect haemocytes: what type of cell is that? J Insect Physiol 52:417-429. https://doi.org/10.1016/J. JINSPHYS.2006.01.005

50. Durrant J, Green MP, Jones TM (2020) Dim artificial light at night reduces the cellular immune response of the black field cricket, Teleogryllus commodus. Insect Sci 27:571-582. https://doi.org/ 10.1111/1744-7917.12665

51. Ziegler A-K, Watson H, Hegemann A et al (2021) Exposure to artificial light at night alters innate immune response in wild great tit nestlings. J ExpBiol 224.https://doi.org/10.1242/JEB.239350

52. Saini C, Hutton P, Gao S et al (2019) Exposure to artificial light at night increases innate immune activity during development in a precocial bird. Comp Biochem Physiol Part A Mol Integr Physiol 233:84-88. https://doi.org/10.1016/J.CBPA.2019.04.002

53. Becker DJ, Singh D, Pan Q et al (2020) Artificial light at night amplifies seasonal relapse of haemosporidian parasites in a widespread songbird. Proc R Soc B 287.https://doi.org/10.1098/RSPB. 2020.1831

54. Ouyang JQ, de Jong M, van Grunsven RHA et al (2017) Restless roosts: light pollution affects behavior, sleep, and physiology in a free-living songbird. Glob Chang Biol 23:4987-4994. https://doi. org/10.1111/GCB.13756

55. Okuliarova M, Mazgutova N, Majzunova M et al (2021) Dim light at night impairs daily variation of circulating immune cells and renal immune homeostasis. Front Immunol 3548. https://doi.org/ 10.3389/FIMMU.2020.614960

56. Oishi K, Shibusawa K, Kakazu H, Kuriyama T, Ohkura N, Machida K (2006) Extended light exposure suppresses nocturnal increases in cytotoxic activity of splenic natural killer cells in rats. Biol Rhythm Res 37(01):21-35. https://doi.org/10.1080/ 09291010500386774

57. Perfilyeva YV, Abdolla N, Ostapchuk YO et al (2017) (2017) Expansion of CD11b+Ly6Ghigh and CD11b+CD49d+ myeloid cells with suppressive potential in mice with chronic inflammation and light-at-night-induced circadian disruption. Inflamm Res 668(66):711-724. https://doi.org/10.1007/S00011-017-1052-4

58. Fonken LK, Haim A, Nelson RJ (2012) Dim light at night increases immune function in nile grass rats, a diurnal rodent. Chronobiol Int 29(1):26-34. https://doi.org/10.3109/07420528. 2011.635831

59. Bedrosian TA, Fonken LK, Walton JC, Nelson RJ (2011) Chronic exposure to dim light at night suppresses immune responses in Siberian hamsters. Biol Lett 7:468-471. https://doi.org/10.1098/ RSBL.2010.1108

60. Cissé YM, Russart K, Nelson RJ (2020) Exposure to dim light at night prior to conception attenuates offspring innate immune responses. PLoS ONE 15:e0231140. https://doi.org/10.1371/ JOURNAL.PONE.0231140

61. Fonken LK, Weil ZM, Nelson RJ (2013) Mice exposed to dim light at night exaggerate inflammatory responses to lipopolysaccharide. Brain Behav Immun 34:159-163. https://doi.org/10. 1016/J.BBI.2013.08.011

62. Chen R, Weitzner AS, McKennon LA, Fonken LK (2021) Light at night during development in mice has modest effects on adulthood behavior and neuroimmune activation. Behav Brain Res 405:113171. https://doi.org/10.1016/J.BBR.2021.113171

63. Fonken LK, Bedrosian TA, Zhang N et al (2019) Dim light at night impairs recovery from global cerebral ischemia. Exp Neurol 317:100-109. https://doi.org/10.1016/J.EXPNEUROL.2019.02. 008

64. Panshikar S, Haldar C (2009) Immune responses of Indian Jungle Bush Quail, P. asiatica, to different photoperiodic regimens during the reproductively inactive phase. Biol Rhythm Res 40:235-247. https://doi.org/10.1080/09291010701875328 
65. Moore CB, Siopes TD (2000) Effects of lighting conditions and melatonin supplementation on the cellular and humoral immune responses in Japanese quail Coturnix coturnix japonica. Gen Comp Endocrinol 119:95-104. https://doi.org/10.1006/GCEN. 2000.7496

66. Kernbach ME, Newhouse DJ, Miller JM et al (2019) Light pollution increases West Nile virus competence of a ubiquitous passerine reservoir species. Proc R Soc B 286.https://doi.org/10.1098/ RSPB.2019.1051

67. Woods GM, Chen YP, Dewar AL et al (2001) Prevention of autoimmunity by induction of cutaneous tolerance. Cell Immunol 207:1-5. https://doi.org/10.1006/CIMM.2000.1733

68. Mizutani H, Tamagawa-Mineoka R, Minami Y et al (2017) Constant light exposure impairs immune tolerance development in mice. J Dermatol Sci 86:63-70. https://doi.org/10.1016/J.JDERM SCI.2016.12.016

69. Park JE, Barbul A (2004) Understanding the role of immune regulation in wound healing. Am J Surg 187:S11-S16. https://doi.org/ 10.1016/S0002-9610(03)00296-4

70. Bedrosian TA, Aubrecht TG, Kaugars KE et al (2013)Artificial light at night alters delayed-type hypersensitivity reaction in response to acute stress in Siberian hamsters.https://doi.org/10. 1016/j.bbi.2013.05.009

71. Aubrecht TG, Weil ZM, Nelson RJ (2014) Dim light at night interferes with the development of the short-day phenotype and impairs cell-mediated immunity in Siberian hamsters (Phodopus sungorus). J Exp Zool Part A Ecol Genet Physiol 321:450-456. https://doi.org/10.1002/JEZ.1877

72. Cissé YM, Russart KLG (2017) Nelson RJ (2017) Parental exposure to dim light at night prior to mating alters offspring adaptive immunity. Sci Rep 71(7):1-10. https://doi.org/10.1038/srep45497

73. Sato S, Sakurai T, Ogasawara J, Takahashi M, Izawa T, Imaizumi K, Taniguchi N, Ohno H, Kizaki T (2014) A circadian clock gene, Rev-erb $\alpha$, modulates the inflammatory function of macrophages through the negative regulation of $\mathrm{Ccl} 2$ expression. J Immunol 192(1):407-417

74. Sugimoto T, Morioka N, Zhang FF, Sato K, Abe H, HisaokaNakashima K, Nakata Y (2014) Clock gene Per1 regulates the production of CCL2 and interleukin-6 through p38, JNK1 and NF- $\mathrm{KB}$ activation in spinal astrocytes. Mol Cell Neurosci 59:37-46

75. Fonken LK, Frank MG, Kitt MM et al (2015) Microglia inflammatory responses are controlled by an intrinsic circadian clock. Brain
Behav Immun 45:171-179. https://doi.org/10.1016/j.bbi.2014.11. 009

76. Vieira E, Mirizio GG, Barin GR, de Andrade RV, Nimer NFS, La Sala L (2020) Clock genes, inflammation and the immune system-implications for diabetes, obesity and neurodegenerative diseases. Int J Mol Sci 21(24):9743

77. Bedrosian TA, Weil ZM, Nelson RJ (2013) Chronic dim light at night provokes reversible depression-like phenotype: possible role for TNF. Mol Psychiatry 18:930-936. https://doi.org/10.1038/mp. 2012.96

78. Hogan MK, Kovalycsik T, Sun Q et al (2015) Combined effects of exposure to dim light at night and fine particulate matter on $\mathrm{C} 3 \mathrm{H} /$ HeNHsd mice. Behav Brain Res 294:81-88. https://doi.org/10. 1016/j.bbr.2015.07.033

79. Bumgarner JR, Walker WH, Liu JA et al (2020) Dim light at night exposure induces cold hyperalgesia and mechanical allodynia in male mice. Neuroscience 434:111-119. https://doi.org/10.1016/j. neuroscience.2020.03.022

80. Okuliarova M, Mazgutova N, Majzunova M et al (2021) Dim light at night impairs daily variation of circulating immune cells and renal immune homeostasis. Front Immunol 11:3548. https://doi. org/10.3389/fimmu.2020.614960

81. Mindel JW, Rojas SL, Kline D et al (2019) Sleeping with low levels of artificial light at night increases systemic inflammation in humans. Sleep 42:A15-A16

82. Raap T, Casasole G, Pinxten R, Eens M (2016) Early life exposure to artificial light at night affects the physiological condition: an experimental study on the ecophysiology of free-living nestling songbirds. Environ Pollut 218:909-914

83. Kriegsfeld LJ, LeSauter J, Hamada T et al (2002) Circadian rhythms in the endocrine system. Horm Brain Behav 33-91.https://doi.org/10.1016/B978-012532104-4/50020-2

84. Nelson RJ, Bumgarner JR, Walker WH, DeVries AC (2021) Timeof-day as a critical biological variable. Neurosci Biobehav Rev 127:740-746. https://doi.org/10.1016/J.NEUBIOREV.2021.05. 017

Publisher's note Springer Nature remains neutral with regard to jurisdictional claims in published maps and institutional affiliations. 